\title{
Cerebrovascular Complications Related to Atrial Fibrillation Ablation and Strategies for Periprocedural Stroke Prevention
}

Zoltan Csanadi, MD, PhD ${ }^{a, b, c, *}$, Edina Nagy-Baló, MD ${ }^{a, b, c}$,

Stephan Danik, MD $D^{b, c, d}$, Conor Barrett, $\mathrm{MD}^{\mathrm{b}, \mathrm{c}, \mathrm{d}}$,

J. David Burkhardt, MD ${ }^{\mathrm{b}, c, \mathrm{e}}$, Javier Sanchez ${ }^{\mathrm{b}, \mathrm{c}, \mathrm{e}}$,

Pasquale Santangeli, MD $D^{b, c, f}$, Francesco Santoro, MD ${ }^{b, c, g}$,

\section{KEYWORDS}

- Atrial fibrillation • Stroke • Silent cerebral ischemia

- Diffusion-weighted cerebral magnetic resonance imaging • Transcranial Doppler

\section{KEY POINTS}

- Manifest, clinical stroke related to ablation of atrial fibrillation occurs in about $1 \%$ of patients.

- Silent cerebral ischemia can be detected by diffusion-weighted magnetic resonance imaging (MRI) in as many as $50 \%$ of patients postablation.

- The long-term significance of these silent lesions is not yet known.

- Postablation diffusion-weighted MRI and intraprocedural transcranial Doppler recordings of cerebral microemboli can be used to compare the thrombogenic potential of different ablation techniques.

- A safe periprocedural strategy using novel oral anticoagulants needs to be determined.

- Prospective randomized trials are needed to establish the optimal postablation care of patients regarding long-term anticoagulation.

\section{INTRODUCTION}

Transcatheter treatment of atrial fibrillation $(\mathrm{AF})$ is a complex intervention requiring the introduction of hardware into the left atrium (LA), energy applications over a large area of the LA endocardium, and prolonged instrumentation in the systemic cir- Q9 culation. $^{1}$ Furthermore, these procedures are

\footnotetext{
The authors have nothing to disclose.

${ }^{a}$ Department of Cardiology, University of Debrecen, 22 Móricz Zs, Debrecen H4032, Hungary; ${ }^{\text {b }}$ Case Western Reserve University, Cleveland, OH, USA; ' Interventional Electrophysiology, Scripps Clinic, San Diego, CA, USA; ${ }^{d}$ Al-Sabah Arrhythmia Institute (AI), St. Luke's Hospital, NY, USA; e Texas Cardiac Arrhythmia Institute, St. David's Medical Center, Austin, TX, USA; ${ }^{f}$ Clinical Cardiac Electrophysiology, University of Pennsylvania, Philadelphia, PA, USA; ${ }^{9}$ Department of Cardiology, University of Foggia, Foggia, Italy; ${ }^{\mathrm{h}}$ Albert Einstein College of Medicine, Montefiore Hospital, Bronx, NY, USA; ${ }^{\text {i }}$ Department of Biomedical Engineering, University of Texas, Austin, TX, USA

* Corresponding author.

E-mail address: drcsanadi@hotmail.com
} 
performed in patients who are at inherently increased risk of a thromboembolic complication, including stroke. It is therefore not surprising that cerebrovascular accidents have been among the most feared complications since the inception of AF ablation, evoking significant concern.

\section{INCIDENCE OF CEREBROVASCULAR COMPLICATIONS RELATED TO AF ABLATION Stroke and Transient Ischemic Attack}

The first worldwide survey on catheter ablation for AF concluded that clinical stroke occurred in $0.28 \%$ and transient ischemic attack (TIA) in $0.66 \%$ of patients. The update of that survey, relating to $\mathrm{AF}$ ablations performed between 2003 and 2006, indicated similar rates of cerebrovascular complications $(0.23 \%$ for stroke, $0.71 \%$ for TIA) despite an apparently more challenging patient population with a more enlarged $\mathrm{LA}$ and more persistent AF. ${ }^{2} \mathrm{~A}$ meta-analysis based on the data of 6936 patients who underwent AF ablation by the end of 2006 found that stroke and TIA occurred in $0.3 \%$ and $0.2 \%$, respectively. ${ }^{3}$ Stroke incidences as high as $5 \%^{4}$ and as low as $0 \%{ }^{5}$ have also been reported as single-center findings. Although the complication rates associated with any procedure, including AF ablation, generally decrease with increasing experience, this was not demonstrated in a high-volume center: while the overall complication rate decreased over a 10 -year period from $11.1 \%$ to $1.6 \%$, the incidence of stroke and TIA remained unchanged. ${ }^{6}$ Thromboembolic events typically occur within 24 hours of the ablation procedure, with the high-risk period extending for 2 weeks thereafter. ${ }^{7}$ Stroke is a significant cause of periprocedural death during AF ablation. An international survey on AF ablation in 162 centers reported details of 32 deaths in 32,569 patients. The fatal outcome was attributed to stroke in 5 (16\%) of these 32 cases. ${ }^{8}$ On the other hand, patients who survive a stroke associated with AF ablation often have a favorable long-term prognosis. During a mean 38-month follow-up of 26 patients who suffered AF ablation-related stroke in a high-volume center (2 patients died), complete long-term functional and neurocognitive recovery was documented in most patients, irrespective of the severity of the periprocedural stroke. $^{9}$

\section{Silent Cerebral Ischemia}

It has recently been recognized that silent cerebral ischemia (SCl) can be demonstrated by diffusionweighted cerebral magnetic resonance imaging (DW-MRI) in a much higher proportion of patients undergoing LA ablation than in those with manifest stroke. ${ }^{10-17}$ Lickfett and colleagues ${ }^{10}$ performed DW-MRI before and after a Lasso-guided pulmonary vein (PV) ostium isolation (PVI), and demonstrated new cerebral lesions in 2 (10\%) of 20 patients without overt clinical symptoms. Similarly, an $11 \%$ incidence of $\mathrm{SCl}$ was reported from the same center in a larger population of $53 \mathrm{pa-}$ tients. ${ }^{11}$ In a large-scale study ${ }^{12}$ of 232 patients undergoing PVI with or without linear lesions and targeting of complex fractionated electrograms (CFE) with irrigated radiofrequency (RF) ablation, new silent brain lesions were found on DW-MRI in 33 patients (14\%). These initial results were followed by several single-center studies ${ }^{13-18}$ that reported widely variable results, including an incidence as high as $50 \%$ for a new SCl depending on the ablation and the MRI technology used (Table 1). A recent study examined the ability of a 3-T MRI scan to detect cerebral injury. Of 22 patients who had undergone PVI using cryoenergy, the incidence of $\mathrm{SCl}$ was $50 \%$ as opposed to $27 \%$ of 15 patients who had undergone PVI using RF energy. ${ }^{17}$

\section{Clinical Relevance of SCI}

The clinical significance of $\mathrm{SCl}$ after $\mathrm{AF}$ ablation is at present uncertain. Deneke and colleagues ${ }^{18}$ repeated DW-MRI 2 to 56 weeks (median 12 weeks) after ablation in 14 patients in whom a total of 50 new-onset, clinically silent white matter lesions were identified within 48 hours after ablation. No lesion with a diameter smaller than $10 \mathrm{~mm}$ could be identified on the repeated MRI even as early as 2 weeks after the ablation, whereas 3 larger lesions $(>10 \mathrm{~mm})$ were still detected. Of note, all of these follow-up lesions demonstrated a reduction in size with no hemorrhagic component in any of them, despite the patients being on oral anticoagulation (OAC). The disappearance or shrinkage of these lesions, although reassuring to some extent, does not imply the full recovery of pathologic alterations in the brain. In an elegant canine model, typical lesions were demonstrated on DW-MRI and fluid-attenuated inversion recovery images after the injection of gaseous and particulate microemboli. ${ }^{19}$ Clear evidence of ischemic injury, including severe endothelial proliferation, moderate glia cell activation, and mild perivascular lymphocytic infiltrate, was present on histopathologic examination of brain specimens, despite the resolution of most lesions on MRI by day 4 postembolism.

In the general population of patients with $\mathrm{AF}, \mathrm{a}$ high prevalence of $\mathrm{SCl}$ has consistently been detected on DW-MRI. These subclinical lesions were linked to an unfavorable long-term clinical 


\begin{tabular}{|c|c|c|c|c|}
\hline Authors, Ref. Year & ACT & $\mathbf{N}$ & Ablation Technique & Positive DW-MRI \\
\hline Lickfett et al, ${ }^{10} 2006$ & $>250$ & 10 & Irrigated RF ablation & $1(10 \%)$ \\
\hline Schwarz et al, ${ }^{13} 2010$ & $>300$ & $\begin{array}{l}13 \\
9 \\
\end{array}$ & Irrigated RF ablation & $3(14.3 \%)$ \\
\hline Neumann et al, ${ }^{14} 2011$ & $>300$ & $\begin{array}{l}44 \\
45 \\
\end{array}$ & $\begin{array}{l}\text { Irrigated RF } \\
\text { Cryoballoon }\end{array}$ & $\begin{array}{l}3(6.8 \%) \\
4(8.9 \%) \\
\end{array}$ \\
\hline Gaita et $\mathrm{al}^{1}{ }^{12} 2010$ & $250-300$ & 232 & Irrigated RF ablation & $33(14 \%)$ \\
\hline Schrickel et al, ${ }^{11} 2010$ & $>250$ & 53 & Irrigated RF ablation & $6(11 \%)$ \\
\hline Herrera Siklódy et al, ${ }^{15} 2011$ & $>300$ & $\begin{array}{l}27 \\
23 \\
24\end{array}$ & $\begin{array}{l}\text { RF } \\
\text { Cryoballoon } \\
\text { Phased RF }\end{array}$ & $\begin{array}{l}2(7.4 \%) \\
1(4.3 \%) \\
8(33 \%)\end{array}$ \\
\hline Gaita et al, ${ }^{16} 2011$ & $>300$ & $\begin{array}{l}36 \\
36 \\
36\end{array}$ & $\begin{array}{l}\text { Irrigated RF ablation } \\
\text { Phased RF } \\
\text { Cryoballoon }\end{array}$ & $\begin{array}{l}3(8.3 \%) \\
14(38.9 \%) \\
2(5.6 \%)\end{array}$ \\
\hline
\end{tabular}

Abbreviations: ACT, activated clotting time; DW-MRI, diffusion-weighted cerebral magnetic resonance imaging; RF, radiofrequency.

outcome, including an impaired cognitive function, an increased risk of dementia, and a worse prognosis of AF-related strokes in comparison with those of non-AF etiology. ${ }^{20,21}$ A recent crosssectional study of 180 patients with variable forms of $\mathrm{AF}$ demonstrated an $82 \%$ (paroxysmal) and a 92\% (persistent $\mathrm{AF}$ ) prevalence of $\mathrm{SCl}$ on $\mathrm{MRI}$; the number of lesions per person was significantly higher in the patients with persistent $\mathrm{AF}$ than in those with paroxysmal AF. ${ }^{22}$ Furthermore, the performance in cognitive function tests was significantly poorer in AF patients compared with matched controls.

However, these observations indicating the clinical significance of spontaneous ischemic lesions in AF patients may not be extrapolated to $\mathrm{SCl}$ induced by AF ablation. Whereas postablation $\mathrm{SCl}$ are attributed to microembolization during or shortly after the procedure, the mechanism in patients with AF of variable duration is likely to be multifactorial, with the potential importance of both progressive atherosclerosis and showers of microemboli from the LA. Limited data are available on the cognitive function after AF ablation. Schwarz and colleagues ${ }^{13}$ compared the results of neurophysiologic tests before and 3 months after PVI in 21 patients and found a poorer neurophysiologic outcome in verbal memory, but no difference in the other 4 cognitive domains evaluated (attention, verbal fluency, executive functioning, and visual memory). A battery of 8 neuropsychological tests was performed in a recent study on 150 patients, including 90 undergoing wide encircling antrum ablation for paroxysmal (60 patients) or persistent (25 patients) AF, 30 patients undergoing ablation for supraventricular tachycardia (SVT), and 30 patients scheduled for ablation of $\mathrm{AF}$ as a matched nonoperative control group. ${ }^{23}$ The results at 90 days after ablation indicated postoperative cognitive dysfunction in $13 \%$ of the paroxysmal AF patents, $20 \%$ of the persistent AF patients, $3 \%$ of the SVT patients, and none in the control group. The only predictor of negative changes was the LA access time. The clinical significance of the subtle changes suggested by these data warrants further exploration.

Although direct evidence is still lacking, it is reasonable to assume that $\mathrm{SCl}$ lesions detected by DW-MRI are indicators of the thromboembolic consequences related to AF ablation; a preventive measure that successfully limits the subclinical event rate will also reduce the risk of manifest stroke. Its relatively high incidence therefore makes $\mathrm{SCl}$ a logical and practical surrogate of clinically overt cerebrovascular events in future clinical studies.

\section{MECHANISM OF PERIPROCEDURAL THROMBOEMBOLIZATION}

The postulated mechanisms of AF ablationrelated cerebral embolization include embolism of particulate debris, thrombus, char, or gas bubbles at the site of the ablation in the LA.

\section{Particulate Debris}

Transseptal puncture may produce particulate debris in 2 different ways:

1. While advancing, the transseptal needle scrapes plastic particles off the inner wall of 
the transseptal dilator, thereby creating embolic material. ${ }^{24}$

2. The coring of cardiac tissue into the tip of the open-ended needle creates a small plug of cardiac tissue regardless of the puncture site and the type of the needle, including RF. ${ }^{25}$

\section{Thrombus}

The generation of thrombi relies on Virchow's triad: endothelial injury, hemodynamic changes (stasis and turbulence), and a hypercoagulable state.

Energy application during ablation injures endothelial cells. When the continuity of the endothelium is interrupted, its natural anticoagulation properties are lost and blood components come into direct contact with subendothelial procoagulant proteins, such as collagen, tissue factor, and von Willebrand factor. Consequently, thrombus formation is initiated through platelet adhesion and activation, and thrombin production. ${ }^{26}$ Thrombi adherent to the endothelium may dislodge spontaneously or as a result of catheter manipulation, mechanical trauma resulting from electric cardioversion, and restoration of atrial contractility in sinus rhythm. ${ }^{12}$ Of importance is that the thrombogenic potential is related to the energy applied: cryoablation has been shown to be less thrombogenic than RF. ${ }^{27}$ The difference is explained by the histologic characteristics of RF lesions and cryolesions. Whereas those produced by the latter are well circumscribed with sharp borders, with sparing of most of the endothelial lining, RF lesions are characterized by intralesional hemorrhage and ragged edges, with a marked endothelial injury. Similarly to RF, all energy sources that ablate through heating, such as the microwave and the laser, carry an increased risk of thrombus formation. ${ }^{26}$

Energy delivery can also lead to embolization through the direct embolization of small myocardial fragments generated by steam-pops. This event is more likely to occur when the tissue is overheated because of the high contact forces and/or the high energy delivered during the ablation. ${ }^{28}$ Char formation at the tip of the catheter in these situations is not uncommon, and may also be a potential source of embolization. ${ }^{29}$

Hemodynamic changes, including stasis and turbulence, may also contribute to thrombus formation during LA ablation. Stasis can occur in the transseptal sheath and in the trapped blood column in the PV behind an occluding cryoballoon, providing the proper milieu for thrombus formation, which may enter the systemic circulation during catheter exchange or deflation of the balloon. ${ }^{12,30}$ The turbulent blood flow created by the catheter manipulation or the rapid injection of contrast material may induce a response that results in platelet activation, which in turn may begin the process ultimately leading to embolization. ${ }^{31}$

A hypercoagulable state develops during PVI through 2 main mechanisms. Heating of the circulating blood elements during RF energy delivery has been demonstrated to activate platelets and the clotting system. ${ }^{32}$ However, the introduction of the catheters and sheaths themselves activates the coagulation cascade and induces a prothrombotic state. ${ }^{33}$ This concept was demonstrated by Ren and colleagues, ${ }^{34}$ who detected fresh thrombi attached to a sheath or mapping catheter by intracardiac echocardiography (ICE) in as many as $10 \%$ of the cases.

\section{Gas Bubbles}

As transseptal sheaths may potentially connect the LA with the room air, flushing through these sheaths, or introducing or exchanging catheters and guide wires, pose the risk of air embolization, even with the protection of hemostatic valves. The risk of air embolization is higher when catheters with a complex configuration are used. ${ }^{35}$ Furthermore, gas embolization can occur during other phases of these procedures, including the energy delivery period, PV angiography, and catheter manipulation. ${ }^{36}$ Microcavitation visualized as bubbles by ICE occurs during RF delivery, especially when the tissue temperature exceeds $60^{\circ} \mathrm{C}^{37}$ The phenomenon of cavitation, well known in stainless-steel turbines, dam outlets, and ship propellers, and first described in a cardiovascular context in connection with mechanical heart valves, involves the rapid formation of vaporous microbubbles in a fluid owing to a local reduction of pressure to below the vapor pressure. $^{38}$

\section{INTRAPROCEDURAL ASSESSMENT OF THROMBOEMBOLIC RISK DURING LA ABLATION}

DW-MRI has become the gold standard for assessment of cerebral ischemia, either symptomatic or asymptomatic, after ablation. A realtime assessment of the thromboembolic risk during the ablation may improve the safety of the procedure. Two methods have been used for this purpose: the monitoring of microbubbles on ICE, and the detection of microembolic signals (MES) in the cerebral arteries by transcranial Doppler (TCD). 


\section{Monitoring of Bubble Formation by ICE} During Ablation; Power Titration Strategy

ICE was originally introduced to interventional electrophysiology as a simple and reliable tool to display different cardiac structures and the positions of catheters in the heart, ${ }^{39}$ to ensure safe transseptal puncture and the early recognition of complications during the procedure. ICEdetectable bubble formation during RF delivery was first described in an experimental model by Kalman and colleagues, ${ }^{37}$ who noticed that showers of microbubbles often preceded an increase in impedance, indicating overheating of tissue during ablation. The concept was first tested in humans by Marrouche and colleagues, ${ }^{40}$ who developed an energy titration strategy based on the microbubble density detected by ICE. Two types of bubble-generation patterns were defined: scattered microbubbles (type 1), indicating early tissue overheating, and a brisk shower of dense microbubbles (type 2) (Fig. 1). The energy was increased in stepwise fashion until the appearance of the type 1 pattern, and the power was then reduced. Energy delivery was immediately terminated in the event of the type 2 pattern. This strategy prevented any thromboembolic complication in 152 patients undergoing circular mappingguided PVI, whereas stroke/TIA occurred in $3 \%$ of patients without ICE-guided power titration. Ablation of complex substrates including AF under ICE guidance with power titration has become a routine practice in many centers, although the assessment of bubble density is compromised by echogenic microbubble formation caused by the irrigation flow when open irrigated catheters are used. A semiquantitative scale describing the

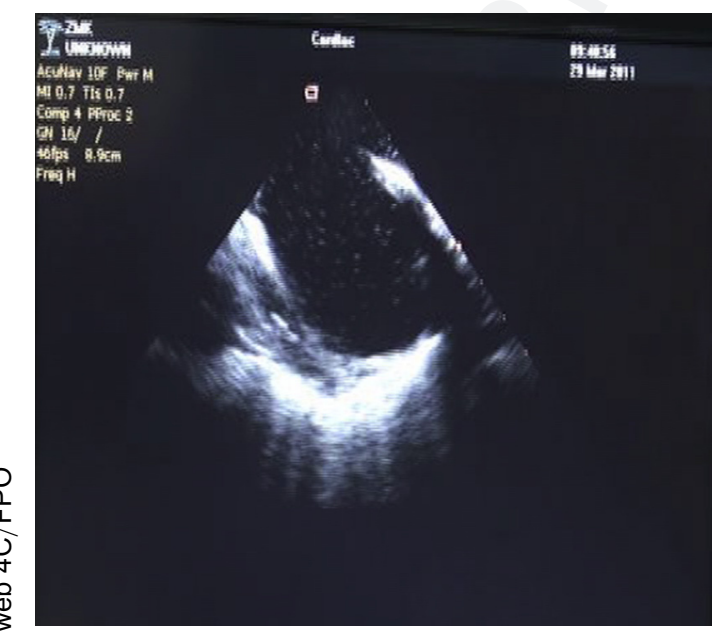

Fig. 1. Microbubbles detected by phased-array intracardiac echocardiography. bubble density of 3 different patterns as few, moderate, or shower has also been used.

Microembolic Signal Detection by Transcranial Doppler

Circulating cerebral emboli can be detected by TCD when imaging the middle cerebral arteries (Fig. 2). MES are characterized by short-term, high-intensity ultrasonic signals with characteristic audible chirps. ${ }^{41,42}$ With older devices, the differentiation between true embolic signals and artifacts (probe dislocation or noise from external devices) requires an experienced observer. MES may be due to solid particles, or gaseous in nature. As there is a difference in their acoustic impedance, solid and gaseous emboli can be differentiated. The latter reflect the ultrasonic beam with a higher intensity than do denser particles. The use of novel multifrequency TCDs with imaging at 2 different frequencies ( 2 and $2.5 \mathrm{MHz}$ ) can automatically differentiate true signals from noise, and gaseous from solid emboli, thereby improving the practicality of the technique for routine clinical use. ${ }^{43}$ Results of several studies have been reported with MES detection during cardiopulmonary bypass surgery and carotid interventions. ${ }^{44}$ The clinical significance of these microemboli with regard to the postoperative neurologic state or the cognitive function of the patients is less well established.

Limited data are available on the number of MES during LA ablation for AF. Kilicaslan and colleagues ${ }^{45}$ compared MES counts recorded during PV antrum isolation with ICE-guided power titration (as described earlier) versus that with conventional power-limited RF delivery in 202 patients. A good correlation was found between the intensity of bubble formation and the MES count. The power titration strategy resulted in half the total number of MES (mean = 1015) in comparison with the conventional approach (mean $=2250$ ), and acute neurologic complications occurred in $0.9 \%$ and $3.1 \%$ of patients, respectively. Sauren and colleagues ${ }^{46}$ reported a virtually negligible number of MES (mean =5) during epicardial AF ablation in comparison with endocardial ablation (mean $=$ 3908). In another study from the same group, ${ }^{30}$ the MES counts detected during AF ablation demonstrated significant differences between 3 different techniques (cryoballoon, irrigated RF, and nonirrigated RF), in line with previous MRI results. Nagy-Baló and colleagues ${ }^{36}$ recently reported on the results of intraoperative TCD and ICE recording in 34 patients undergoing $\mathrm{PVI}$ with either cryoballoon or phased-RF ablation. It is noteworthy that multifrequency TCD capable of 

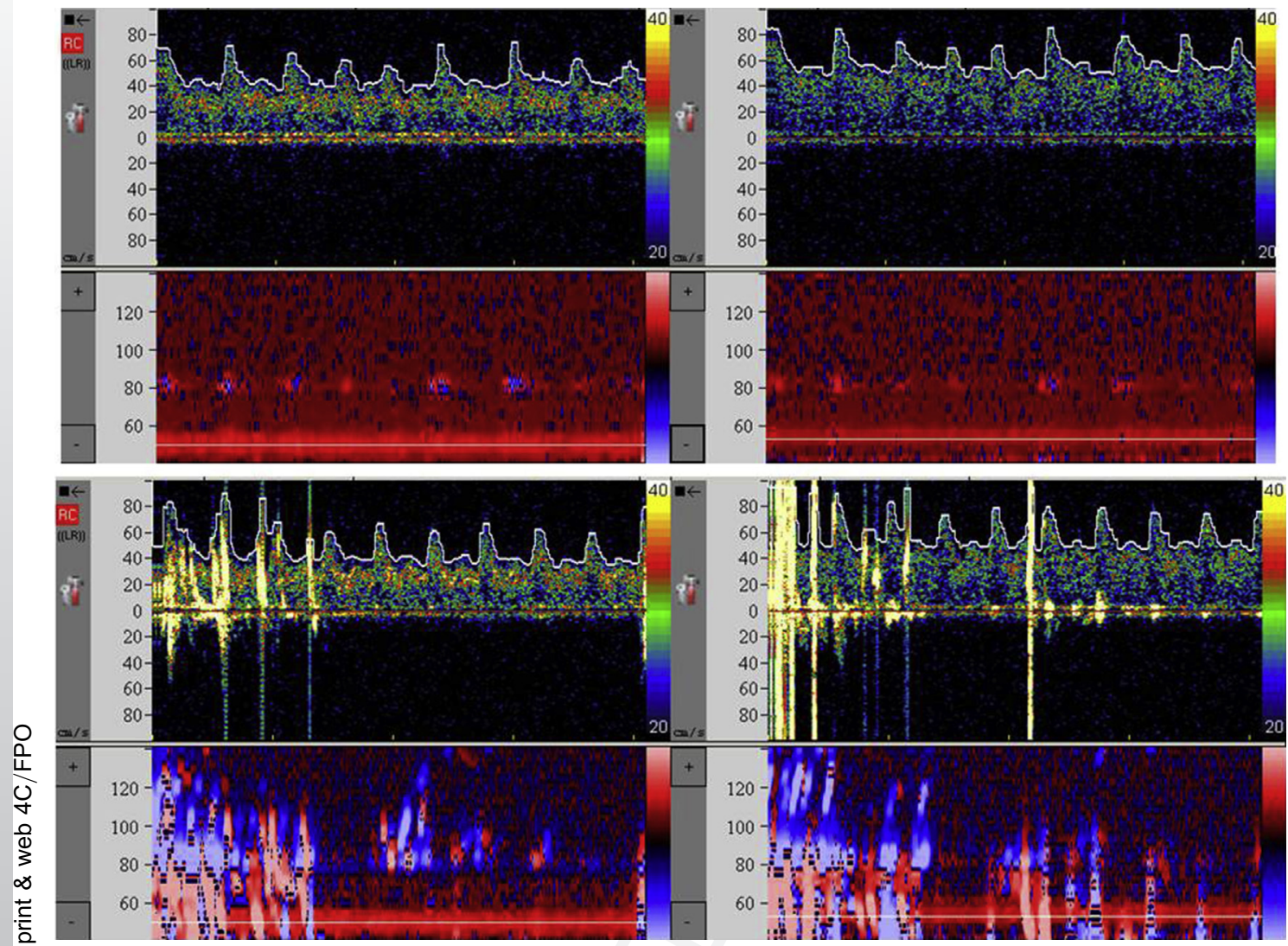

Fig. 2. Bilateral transcranial Doppler recording of microembolic signals (MES) during radiofrequency ablation in the left atrium. MES can be observed on the lower but not on the upper panels.

automatic differentiation of gaseous and solid emboli was used to study the nature of the MES. A very significant correlation was demonstrated between the microbubble density and the MES count, confirming previous observations. ${ }^{45}$ In line with published DW-MRI results, significantly lower total numbers of MES were detected during cryoballoon ablation in comparison with phased-RF ablation. This study was the first to investigate the nature of the MES, and demonstrated that $80 \%$ of them were of gaseous origin regardless of the ablation technique. The significance of the composition of the microemboli at this time is unclear. In theory, gaseous bubbles are expected to be less durable and less harmful than particles. With no data available on the relationship between the MES count recorded during ablation and postprocedural DW-MRI findings or manifest cerebral ischemia, it is not possible even to estimate the microembolic load that would indicate a significant risk of a symptomatic or an MRI-detectable lesion. However, MES detection promises to be a valuable tool to compare the thromboembolic potentials of different ablation techniques and strategies, and to gain further insight into the mechanisms of embolus formation relating to different stages of AF ablation.

\section{CLINICAL AND ABLATION TECHNOLOGY- RELATED PREDICTORS OF CEREBRAL VASCULAR EVENTS}

The risk of a periprocedural cerebral ischemic event, either symptomatic or subclinical, is influenced by multiple factors, including the baseline characteristics of patients and the technical aspects of the ablation procedure.

\section{Patients' Characteristics}

In a prospective multicenter study ${ }^{47}$ on 6454 patients undergoing RF ablation for $\mathrm{AF}$ in 9 centers, stroke/TIA occurred in 27 patients (1.1\%). Among the characteristics of the cohort, diabetes mellitus, congestive heart failure, and the type of $\mathrm{AF}$ (paroxysmal or nonparoxysmal) proved to be independent predictors of a periprocedural cerebrovascular event on multivariate analysis. In a single-center study, ${ }^{48} 10(1.4 \%)$ of 721 patients 
suffered a stroke/TIA during or within 30 days after AF ablation, and a CHADS2 score of 2 or higher and a history of previous stroke/TIA remained independent predictors of cerebral ischemia in 2 multivariate models.

Inconsistent DW-MRI results have been published regarding clinical predictors of SCl. In the largest population studied so $\mathrm{far}^{12}{ }^{12}$ none of the clinical characteristics were predictive of $\mathrm{SCl}$. Schrickel and colleagues ${ }^{11}$ reported 6 new cases of $\mathrm{SCl}$ in 53 patients after focal RF ablation. Coronary artery disease; the number of failed antiarrhythmic drugs, an enlarged left ventricular volume, and septal-wall thickness were predictors of a positive DW-MRI finding. A Japanese study ${ }^{49}$ found only left ventricular ejection fraction to be a positive predictor. In the MEDAFI trial, ${ }^{14}$ which compared cryoablation and irrigated RF ablation (nonrandomized) in 89 patients, age was the only predictor of $\mathrm{SCl}$.

\section{Technical Aspects of the Ablation Procedure}

Besides the ablation technology, consideration must also be given to the manipulation of the sheaths placed in the LA, and the types of energy and catheter used for the ablation.

Long sheaths, including those used to establish LA access during transseptal puncture and steerable sheaths designed to facilitate maneuvering in the LA, pose well-known hazards of air embolism during injections or flushing through these devices, and also during catheter exchange, as removal of the catheter can create a vacuum inside the lumen. These devices, mostly at their distal segment, are a source of thrombus formation, especially in the absence of appropriate and timely anticoagulation (Fig. 3). Continuous flushing

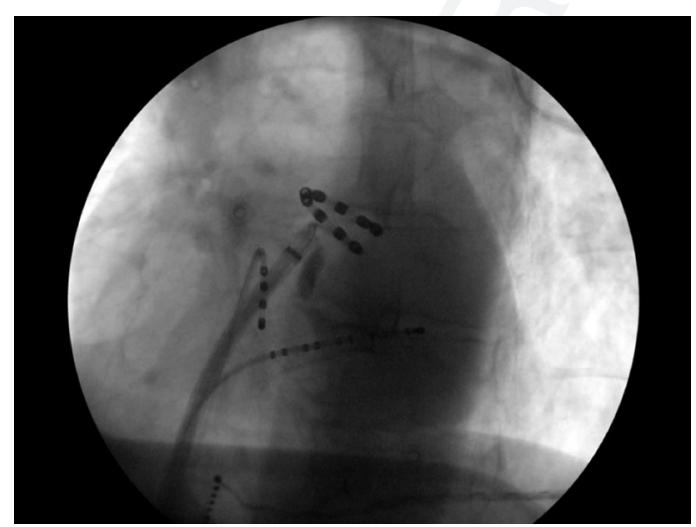

Fig. 3. Thrombus formation on the tip of a steerable sheath in the left atrium. Thrombus attached to the tip of the sheath is visible on fluoroscopy after contrast injection. of these sheaths and meticulous care to eliminate air bubbles are essential for procedure safety. ${ }^{50}$ Furthermore, any catheter removal should be performed slowly with continuous suction on the side arm of the sheaths, followed by careful flushing. It is a common practice in many centers to keep the sheaths in the right atrium once the diagnostic and ablation catheters have been placed in the $L A$, thereby mitigating the risk and consequences of thrombus formation on the tip of the sheath. Besides other advantages, the routine use of ICE offers an opportunity for the continuous monitoring of all catheters placed in the heart, with the recognition of thrombus formation on them. ${ }^{34}$

The ablation technology, including the type of energy and the catheter used for AF ablation, can lead to different risks. Since the early days of AF ablation, when RF energy was used exclusively with conventional $4-\mathrm{mm}$ and then 8-mm tip ablation catheters, alternative energy sources including cryoenergy and laser have been introduced. In the present era, irrigation RF catheters have become the standard, and balloon-based and multipolar ablation technologies are popular in many centers. It is reasonable to assume that significant differences in thromboembolic risk may be associated with these different technologies. However, conclusive evidence as to the advantage of one technology over another, measured as a difference in the rates of manifest stroke/TIA, is not yet available, largely because of the relatively low occurrence of clinically overt events.

The much higher incidence of SCl events offers a better opportunity for the comparison of these ablation methods. In fact, significant differences have been demonstrated in the rate of SCI. Gaita and colleagues ${ }^{16}$ reported a striking ablation technology-dependent difference in the incidence of $\mathrm{SCl}$ detected by MRI. In a randomized comparison of 108 patients, phased-RF ablation with a pulmonary vein ablation catheter (PVAC) was associated with a significantly higher (38.9\%) incidence of acute lesions than was observed with irrigated focal ablation (8.3\%) or cryoablation (5.6\%). In 74 patients, Herrera Siklódy and colleagues $^{15}$ found new ischemia on DW-MRI in $37.5 \%$ after PVAC ablation, compared with $7.4 \%$ and $4.3 \%$ after irrigated RF and cryoablation, respectively. In a recent report, ${ }^{51}$ the use of phased-RF ablation with a new generator and modified software to control power handling during RF applications decreased the incidence of positive findings on DW-MRI to $27 \%$. Another publication by the same group ${ }^{52}$ indicated that simultaneous RF delivery to no more than 2 electrode pairs and exclusion of the first and the last 
poles from the simultaneous energy application further reduced the incidence of SCI to $11.7 \%$, at the price of a prolonged procedure time.

Although it seems reasonable to assume that the procedure (and/or the LA) time and the amount of ablation with the addition of lines or atrial defragmentation may also influence the thromboembolic risk, this has not been confirmed. ${ }^{12,49}$ However, coronary angiography performed together with $\mathrm{AF}$ ablation ${ }^{49}$ and cardioversion (either pharmacologic or electrical) during ablation ${ }^{12}$ have been found to be positive predictors.

The available data on the number of MES detected by TCD during AF ablation also demonstrate marked technology-dependent differences (Table 2). Sauren and colleagues ${ }^{30}$ observed significantly more MES during ablation with nonirrigated $\mathrm{RF}$ (mean $=3908$ ) compared with irrigated RF (1404) and cryoablation (935). In a recent study, Nagy-Baló and coleagues ${ }^{36}$ compared the number of MES during cryoablation and PVAC ablation using lower $(\geq 250)$ and higher $(\geq 320)$ minimum intraprocedural activated clotting time (ACT) target levels for PVAC. Irrespective of the level of anticoagulation, ablation with the PVAC (means $=3143$ and 2205 ) resulted in significantly higher MES counts in comparison with cryoablation (mean $=834$ ).

\section{PERIPROCEDURAL THROMBOEMBOLISM PROPHYLAXIS AND LONG-TERM ANTICOAGULATION AFTER AF ABLATION Thromboembolism Prophylaxis Before Ablation}

Many patients undergoing AF ablation are at an elevated risk of a thromboembolic complication, and therefore require oral anticoagulation with a vitamin $\mathrm{K}$ antagonist (VKA) or a novel oral anticoagulant (NOAC) according to recent guidelines. ${ }^{53,54}$ The 2012 expert consensus statement specified that all patients who have been in AF for 48 hours or longer or for an unknown duration need effective anticoagulation for at least 3 weeks before the procedure, or should undergo transesophageal echocardiography (TEE) to exclude LA thrombus. ${ }^{50}$ The common practice in the past was bridging. The VKA was discontinued and changed to low molecular weight heparin (LMWH) a few days before the procedure, then switched back afterward. Performing AF ablation on a therapeutic level of anticoagulation (international normalized ratio [INR] 2-3.5) has recently evolved as the preferred approach in many centers, after several studies demonstrated its safety. ${ }^{55,56}$ In fact, the risk of both thromboembolism and bleeding was reduced. With the recent introduction and rapid adoption of NOACs for thromboembolism prophylaxis in AF patients, a new strategy for the perioperative management of these patients is urgently needed. Comparison of uninterrupted warfarin and the direct thrombin inhibitor dabigatran, based on data from a multicenter prospective registry, ${ }^{57}$ indicated significantly higher rates of major bleeding and the composite of bleeding and thromboembolic complications $(6 \%$ and $16 \%)$ with dabigatran in comparison with warfarin (1\% and $6 \%)$. All patients with thromboembolic complications who were on dabigatran had nonparoxysmal $\mathrm{AF}$ and more extensive LA ablation. It should be noted that dabigatran was suspended at least 12 hours (mean 16 hours) before the procedure, and was restarted within 3 hours after hemostasis. In another study, ${ }^{58}$ dabigatran was used in 123 patients with paroxysmal AF with no bleeding or

Abbreviations: MES, microembolic signal; PVAC, pulmonary vein ablation catheter. 
thromboembolic complications. However, in this study dabigatran was discontinued 5 days before ablation, a lower intraprocedural ACT target was set, and LMWH was started immediately after the procedure, with dabigatran being resumed 22 hours later. Dabigatran is known to intensify the effect of unfractionated heparin (UFH) on the activated partial thromboplastin time in vitro. As UFH is administered during ablation, this drugdrug interaction may increase the bleeding risk unless dabigatran is withdrawn at least 1 to 2 days in advance. Therefore, starting dabigatran 3 hours after the procedure, with another 2 to 3 hours required for it to reach its full anticoagulation effect, results in an unprotected time window of 5 to 6 hours during the early postablation period unless LMWH is used for bridging.

One study ${ }^{59}$ examined the use of interrupted rivaroxaban $(n=321)$ in comparison with warfarin $(n=321)$ for patients undergoing PVI. Fifty-one percent of patients in both groups had paroxysmal AF. The respective rates of major bleeding $(1.6 \%$ vs $1.9 \%)$ and embolic events $(0.3 \%$ vs $0.3 \%$ ) were not significantly different between those on rivaroxaban and those on warfarin. Further studies using rivaroxaban, as well as other NOACs (apixaban), will be needed to determine whether these agents offer a safer alternative for periprocedural anticoagulation without the need for bridging.

\section{Preablation Exclusion of LA Thrombus}

The presence of a thrombus in the LA should be excluded before ablation in all patients unless adequate anticoagulation is documented for at least 3 weeks before the procedure. However, it is the routine in many centers for all patients to undergo an evaluation shortly before the ablation, regardless of the presenting rhythm and previous anticoagulation. The gold-standard method is TEE. Multidetector computed cardiac tomography (CCT) has also been proposed to assess the LA appendage for thrombus, but good sensitivity was associated with poor specificity in several reports. ${ }^{60}$ In recent publications, ${ }^{61,62}$ further assessment with delayed CCT at 1 or 3 minutes improved the specificity and positive predictive value to $100 \%$. In many centers, CCT is part of the routine preprocedure; because the CCT data are used for image integration with the electroanatomic mapping system, patient discomfort and the additional costs related to the TEE could be avoided by extending its use to exclude a preexisting thrombus. ICE has also been advocated as a supplementary tool to assess LA thrombus in the event of an equivocal TEE finding. ${ }^{63}$

\section{Intraprocedural Heparin Administration}

Intraprocedural anticoagulation involves UFH administered as an intravenous bolus followed by continuous infusion to maintain a target ACT. As thrombus can build up on the transseptal sheath within a very short time, after or even before crossing the septum, it has become a common practice to give the bolus before or immediately after the transseptal puncture. ${ }^{64}$ The recommended ACT target is 300 to 400 seconds; some centers aim at a level of more than 350 seconds. The target ACT should be reached before the first energy delivery, and then checked regularly (every 20-30 minutes) throughout the procedure. Extra boluses of UFH should be given if the ACT level drops below the target value. UFH administration is discontinued once all catheters and sheaths have been withdrawn from the LA; sheaths from the groin can be removed at an ACT level lower than 200 seconds. Heparin is reversed with protamine in some centers at the end of the procedure.

\section{Postablation Anticoagulation}

Both theoretical considerations and clinical observations recommend oral anticoagulation for at least 2 months for all patients after AF ablation. A variable degree of atrial stunning, similar to that occurring with direct-current cardioversion, is present after AF ablation. Moreover, the fresh endothelial damage resulting from energy application in the LA itself is thrombogenic. It has been demonstrated that most post-AF ablation strokes occur within 2 weeks after the procedure. ${ }^{7}$ In patients undergoing the ablation with therapeutic INR, VKA should be continued for at least 2 months according to current recommendations..$^{50}$ In those managed with the bridging strategy, LMWH should be restarted after sheath removal and continued until the therapeutic INR is reached. Alternatively, oral anticoagulation can be followed by an NOAC.

With no evidence from large-scale randomized trials regarding the long-term thromboembolic risk in these patients, the same therapeutic principles as in patients without ablation are to be applied. The decision should therefore be based on the CHADS2 or CHADS2-VASC score, ${ }^{53,54,65}$ and not on the presence or type of AF after ablation. The prognostic value of these scores after ablation has recently been demonstrated. ${ }^{66}$ The available, but as yet insufficient, data suggest that successful AF ablation does reduce the stroke risk, and that long-term antithrombotic prophylaxis may not be required in all patients. In a nonrandomized study 755 patients were followed for a mean of 25 months postablation. Late thromboembolic events were noted in 2 of 755 patients 
(0.3\%), both of whom were on OAC. ${ }^{7}$ No cerebral complication occurred in 180 patients who had at least 1 risk factor for stroke, remained in sinus rhythm, and in whom anticoagulant therapy was stopped a median of 5 months postablation. In a recent observational study ${ }^{67}$ on 3344 patients who underwent $\mathrm{AF}$ ablation in 5 centers, OAC was discontinued and aspirin was prescribed, regardless of the CHADS2 score, in those who had no recurrence of an atrial tachyarrhythmia or a severe LA mechanical dysfunction. In those with a CHADS2 score of 1 or more, anticoagulation was restarted in the event of recurrence of atrial arrhythmia. In 347 patients with a CHADS2 score of greater than 2, no thromboembolic events were observed during a mean follow-up of 28 months. Bleeding complications were significantly more frequent among those on chronic anticoagulant therapy.

\section{SUMMARY}

While improvements have been made to limit the incidence of thromboembolic events, especially stroke, during catheter ablation of $\mathrm{AF}$, the optimal strategy to minimize such complications has yet to be determined. Although operator experience certainly plays a role in limiting the incidence of stroke, periprocedural anticoagulation strategies that minimize both bleeding and stroke in a standardized fashion have yet to be universally agreed upon. It is hoped that larger trials can be undertaken to definitively address these important concerns.

\section{REFERENCES}

1. Cappato R, Calkins H, Chen SA, et al. Worldwide survey on the methods, efficacy, and safety of catheter ablation for human atrial fibrillation. Circulation 2005;111:1100.

2. Cappato R, Calkins H, Chen SA, et al. Updated worldwide survey on the methods, efficacy, and safety of catheter ablation for human atrial fibrillation. Circ Arrhythm Electrophysiol 2010;3:32-8.

3. Calkins H, Reynolds MR, Spector P, et al. Treatment of atrial fibrillation with antiarrhythmic drugs or radiofrequency ablation: two systematic literature reviews and meta-analyses. Circ Arrhythm Electrophysiol 2009;2:349-61.

4. Kok LC, Mangrum JM, Haines DE, et al. Cerebrovascular complication associated with pulmonary vein ablation. J Cardiovasc Electrophysiol 2002; 13:764-7.

5. Lee G, Sparks PB, Morton JB, et al. Low risk of major complications associated with pulmonary vein antral isolation for atrial fibrillation: results of 500 consecutive ablation procedures in patients with low prevalence of structural heart disease from a single center. J Cardiovasc Electrophysiol 2011; 22:163-8.

6. Hoyt H, Bhonsale A, Chilukuri K, et al. Complications arising from catheter ablation of atrial fibrillation: temporal trends and predictors. Heart Rhythm 2011;8(12):1869-74.

7. Oral H, Chugh A, Özaydin M, et al. Risk of thromboembolic events after percutaneous left atrial radiofrequency ablation of atrial fibrillation. Circulation 2006;114:759-65.

8. Cappato R, Calkins H, Chen SA, et al. Prevalence and causes of fatal outcome in catheter ablation of atrial fibrillation. J Am Coll Cardiol 2009;53: 1798-803.

9. Patel D, Bailey SM, Furlan AJ, et al. Long-term functional and neurocognitive recovery in patients who had an acute cerebrovascular event secondary to catheter ablation for atrial fibrillation. J Cardiovasc Electrophysiol 2010;21:412-7.

10. Lickfett L, Hackenbroch M, Lewalter T, et al. Cerebral diffusion-weighted magnetic resonance imaging: a tool to monitor the thrombogenicity of left atrial catheter ablation. J Cardiovasc Electrophysiol 2006;17:1-7.

11. Schrickel JW, Lickfett L, Lewalter T, et al. Incidence and predictors of silent cerebral embolism during pulmonary vein catheter ablation for atrial fibrillation. Europace 2010;12:52-7.

12. Gaita F, Caponi D, Pianelli M, et al. Radiofrequency catheter ablation of atrial fibrillation: a cause of silent thromboembolism? Magnetic resonance imaging assessment of cerebral thromboembolism in patients undergoing ablation of atrial fibrillation. Circulation 2010;122:1667-73.

13. Schwarz $N$, Kuniss $M$, Nedelmann $M$, et al. Neuropsychological decline after catheter ablation of atrial fibrillation. Heart Rhythm 2010; 7(12):1761-7.

14. Neumann T, Kuniss M, Conradi G, et al. MEDAFItrial (micro-embolization during ablation of atrial fibrillation): comparison of pulmonary vein isolation using cryoballoon technique vs. radiofrequency energy. Europace 2011;13:37-44.

15. Herrera Siklódy C, Deneke T, Hocini M, et al. Incidence of asymptomatic embolic events following pulmonary vein isolation procedures: comparison between different ablation devices. J Am Coll Cardiol 2011;58:681-8.

16. Gaita F, Leclercq JF, Schumacher B, et al. Incidence of silent cerebral thromboembolic lesions after atrial fibrillation ablation may change according to technology used: comparison of irrigated radiofrequency, multipolar nonirrigated catheter and cryoballoon. J Cardiovasc Electrophysiol 2011;22: 961-8.
1049

1050

1051

1052

1053

1054

1055

1056

1057

1058

1059

1060

1061

1062

1063

1064

1065

1066

1067

1068

1069

1070

1071

1072

1073

1074

1075

1076

1077

1078

1079

1080

1081

1082

1083

1084

1085

1086

1087

1089

1090

1091

1092

1093

1094

1095

1096

1097

1098

1099

1100

1101

1102

1103

1104

1105 
17. Haeusler KG, Koch L, Herm J, et al. 3 Tesla MRI-detected brain lesions after pulmonary vein isolation for atrial fibrillation: results of the MACPAF study. J Cardiovasc Electrophysiol 2013;24(1):14-21.

18. Deneke T, Shin DI, Balta O, et al. Post-ablation asymptomatic cerebral lesions-long-term followup using magnetic resonance imaging. Heart Rhythm 2011;8:1705-11.

19. Haines DE, Stewart MT, Barka ND, et al. Microembolism and catheter ablation II: effects of cerebral microemboli injections in a canine model. Circ Arrhythm Electrophysiol 2013;6:23-30.

20. Kalantarian S, Stern TA, Mansour M, et al. Cognitive impairment associated with atrial fibrillation: a meta-analysis. Ann Intern Med 2013;158(5 Pt 1): $338-46$

21. Vermeer SE, Prins ND, den Heier T, et al. Silent brain infarcts and the risk of dementia and cognitive decline. N Engl J Med 2013;348:1215-22.

22. Gaita F, Corsinovi L, Anselmino M, et al. Prevalence of silent cerebral ischemia in paroxysmal and persistent atrial fibrillation and correlation with cognitive function. J Am Coll Cardiol 2013. http:// dx.doi.org/10.1016/jacc2013.05.074.

23. Medi C, Evered L, Silbert B, et al. Subtle postprocedural cognitive dysfunction after atrial fibrillation ablation. J Am Coll Cardiol 2013;62:531-9.

24. Feld GK, Tiongson J, Oshodi G. Particle formation and risk of embolization during transseptal catheterization: comparison of standard transseptal needles and a new radiofrequency transseptal needle. J Interv Card Electrophysiol 2011;30:31-6.

25. Greenstein E, Passman R, Lin AC, et al. Knight incidence of tissue coring during transseptal catheterization when using electrocautery and a standard transseptal needle. Circ Arrhythm Electrophysiol 2012;5(2):341-4.

26. Zhou L, Keane D, Reed G, et al. Thromboembolic complications of cardiac radiofrequency catheter ablation: a review of the reported incidence, pathogenesis and current research directions. J Cardiovasc Electrophysiol 1999;10(4):611-20.

27. Khairy P, Chauvet $P$, Lehmann J, et al. Lower incidence of thrombus formation with cryoenergy versus radiofrequency catheter ablation. Circulation 2003;107(15):2045-50.

28. Di Biase L, Natale A, Barrett C, et al. Relationship between catheter forces, lesion characteristics, "popping," and char formation: experience with robotic navigation system. J Cardiovasc Electrophysiol 2009;20(4):436-40.

29. Nath S, DiMarco JP, Haines DE. Basic aspects of radiofrequency catheter ablation. J Cardiovasc Electrophysiol 1994;5(10):863-76.

30. Sauren LD, Van Belle Y, De Roy L, et al. Transcranial measurement of cerebral microembolic signals during endocardial pulmonary vein isolation: comparison of 3 different ablation techniques. J Cardiovasc Electrophysiol 2009;20(10):1102-7.

31. Nesbitt WS, Mangin P, Salem HH, et al. The impact of blood rheology on the molecular and cellular events underlying arterial thrombosis. J Mol Med 2006;84:989-95.

32. Van Oeveren W, Crijns HJ, Korteling BJ, et al. Blood damage, platelet and clotting activation during application of radiofrequency or cryoablation catheters: a comparative in vitro study. J Med Eng Technol 1999;23(1):20-5.

33. Dorbala S, Cohen AJ, Hutchinson LA, et al. Does radiofrequency ablation induce a prethrombotic state? Analysis of coagulation system activation and comparison to electrophysiologic study. J Cardiovasc Electrophysiol 1998;9(11): 1152-60.

34. Ren JF, Marchlinski FE, Callans DJ. Left atrial thrombus associated with ablation for atrial fibrillation: identification with intracardiac echocardiography. J Am Coll Cardiol 2004;43:1861-7.

35. Haines DE, Stewart MT, Ahlberg S, et al. Microembolism and catheter ablation I: a comparison of irrigated radiofrequency and multielectrode-phased radiofrequency catheter ablation of pulmonary vein ostia. Circ Arrhythm Electrophysiol 2013;6: 16-22.

36. Nagy-Baló E, Tint D, Clemens M, et al. Transcranial measurement of cerebral microembolic signals during pulmonary vein isolation: a comparison of two ablation techniques. Circ Arrhythm Electrophysiol 2013;6:473-80.

37. Kalman JM, Fitzpatrick AP, Olgin JE, et al. Biophysical characteristics of radiofrequency lesion formation in vivo: dynamics of catheter tip-tissue contact evaluated by intracardiac echocardiography. Am Heart J 1997;133(1):8-18.

38. Johansen P. Mechanical heart valve cavitation. Expert Rev Med Devices 2004;1(1):95-104.

39. Szili-Torok T, Kimman GP, Theuns D, et al. Visualisation of intracardiac structures and radiofrequency lesions using intracardiac echocardiography. Eur J Echocardiogr 2003;4: 17-22.

40. Marrouche NS, Martin DO, Wazni O, et al. Phasedarray intracardiac echocardiography monitoring during pulmonary vein isolation in patients with atrial fibrillation: impact on outcome and complications. Circulation 2006;107:2710-6.

41. Ringelstein EB, Droste DW, Babikian WL, et al. Consensus on microembolus detection by TCD. International consensus group on microembolus detection. Stroke 1998;29:725-9.

42. Grosset DG, Georgiadis G, Kelman AW, et al. Detection of microemboli by transcranial Doppler ultrasound. Tex Heart Inst J 1996;23:289-92. 
43. Russell D, Brucher R. Online automatic discrimination between solid and gaseous cerebral microemboli with the first multifrequency transcranial Doppler. Stroke 1998;29:725-9.

44. Borger MA, Peniston CM, Weisel RD, et al. Neurophysiologic impairment after coronary bypass surgery: effect of gaseous microemboli during perfusionist interventions. J Thorac Cardiovasc Surg 2001;121:743-9.

45. Kilicaslan F, Verma A, Saad E, et al. Transcranial Doppler detection of microembolic signals during pulmonary vein antrum isolation: implications for titration of radiofrequency energy. J Cardiovasc Electrophysiol 2006;17:495-501.

46. Sauren LD, La Meir M, de Roy L, et al. Increased number of cerebral emboli during percutaneous endocardial pulmonary vein isolation versus a thoracoscopic epicardial approach. Eur J Cardiothorac Surg 2009;36:833-7.

47. Di Biase L, Burkhardt JD, Mohanty P, et al. Periprocedural stroke and management of major bleeding complications in patients undergoing catheter ablation of atrial fibrillation: the impact of periprocedural therapeutic international normalized ratio. Circulation 2010;121:2550-6.

48. Scherr D, Sharma K, Dalal D, et al. Incidence and predictors of periprocedural cerebrovascular accident in patients undergoing catheter ablation of atrial fibrillation. J Cardiovasc Electrophysiol 2009;20(12):1357-63.

49. Ichiki $\mathrm{H}$, Oketani $\mathrm{N}$, Ishida $\mathrm{S}$, et al. Incidence of asymptomatic cerebral microthromboembolism after atrial fibrillation ablation guided by complex fractionated atrial electrogram. J Cardiovasc Electrophysiol 2012;23(6):567-73.

50. Calkins H, Kuck KH, Cappato R, et al. 2012 HRS/ EHRA/ECAS expert consensus statement on catheter and surgical ablation of atrial fibrillation: recommendations for patient selection, procedural techniques, patient management and follow-up, definitions, endpoints, and research trial design. Heart Rhythm 2012;9(4):632-89.

51. Wieczorek M, Lukat M, Hoeltgen R, et al. Investigation into causes of abnormal cerebral MRI findings following PVAC duty cycled phased RF ablation of atrial fibrillation. J Cardiovasc Electrophysiol 2013; 24(2):121-8.

52. Wieczorek M, Hoeltgen R, Brueck M. Does the number of simultaneously activated electrodes during phased RF multielectrode ablation of atrial fibrillation influence the incidence of silent cerebral microembolism? Heart Rhythm 2013;10(10):953-9.

53. American College of Cardiology Foundation, American Heart Association, European Society of Cardiology, Heart Rhythm Society, Wann LS, Curtis $A B$, Ellenbogen KA, et al. Management of patients with atrial fibrillation (Compilation of
2006 ACCF/AHA/ESC and 2011 ACCF/AHA/HRS Recommendations): a report of the American College of Cardiology/American Heart Association Task Force on Practice Guidelines. Circulation 2013;127:1916-26.

54. European Heart Rhythm Association, European Association for Cardio-Thoracic Surgery, Camm AJ, Kirchhof P, Lip GY, et al. Guidelines for the management of atrial fibrillation. The Task Force for the Management of Atrial Fibrillation of the European Society of Cardiology. Eur Heart J 2010;31: 2369-429.

55. Wazni OM, Beheiry S, Fahmy T, et al. Atrial fibrillation ablation in patients with therapeutic international normalized ratio: comparison of strategies of anticoagulation management in the periprocedural period. Circulation 2007;116:2531-4.

56. Hussein AA, Martin DO, Patel D, et al. Radiofrequency ablation of atrial fibrillation under therapeutic international normalized ratio: a safe and efficacious periprocedural anticoagulation strategy. Heart Rhythm 2009;6:1425-9.

57. Lakkireddy D, Reddy YM, Di Biase L, et al. Feasibility and safety of dabigatran versus warfarin for periprocedural anticoagulation in patients undergoing radiofrequency ablation for atrial fibrillation. results from a multicenter prospective registry. J Am Coll Cardiol 2012;59:1168-74.

58. Winkle RA, Mead RH, Engel G, et al. The use of dabigatran immediately after atrial fibrillation ablation. J Cardiovasc Electrophysiol 2011;23:264-8.

59. Lakkireddy D, Reddy YM, Vallakati A, et al. Feasibility and safety of uninterrupted rivaroxaban for periprocedural anticoagulation in patients undergoing radiofrequency ablation for atrial fibrillation: results from a multicenter prospective registry. J Am Coll Cardiol, in press.

60. Romero J, Husain SA, Kelesidis I, et al. Detection of left atrial appendage thrombus by cardiac computed tomography in patients with atrial fibrillation: a meta-analysis. Circ Cardiovasc Imaging 2013;6(2):185-94.

61. Sawit ST, Garcia-Alvarez A, Suri B, et al. Usefulness of cardiac computed tomographic delayed contrast enhancement of the left atrial appendage before pulmonary vein ablation. Am J Cardiol 2012;109:677-84.

62. Hur J, Pak HN, Kim YJ, et al. Dual-enhancement cardiac computed tomography for assessing left atrial thrombus and pulmonary veins before radiofrequency catheter ablation for atrial fibrillation. Am J Cardiol 2013;112:238-44.

63. Ren JF, Marchlinski FE, Supple GE, et al. Intracardiac echocardiographic diagnosis of thrombus formation in the left atrial appendage: a complementary role to transesophageal echocardiography. Echocardiography 2013;30:72-80. 
64. Bruce CJ, Friedman PA, Narayan O, et al. Early heparinization decreases the incidence of left atrial thrombi detected by intracardiac echocardiography during radiofrequency ablation for atrial fibrillation. J Interv Card Electrophysiol 2008;22:211-9.

65. Camm AJ, Lip GY, De Caterina R, et al. 2012 focused update of the ESC guidelines for the management of atrial fibrillation. Eur Heart J 2012;33: 2719-47.
66. Chao TF, Lin YJ, Tsao HM, et al. CHADS2 and CHA2DS2-VASc scores in the prediction of clinical outcomes in patients with atrial fibrillation after catheter ablation. J Am Coll Cardiol 2011;58: 2380-5.

67. Themistoclakis S, Corrado A, Marchlinski FE, et al. The risk of thromboembolism and need for oral anticoagulation after successful atrial fibrillation ablation. J Am Coll Cardiol 2010;55:735-43. 


\section{AUTHOR QUERY FORM}

\begin{tabular}{|c|c|}
\hline 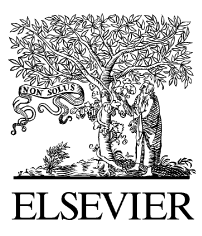 & $\begin{array}{l}\text { Journal: CCEP } \\
\text { Article Number: } 320\end{array}$ \\
\hline
\end{tabular}

Dear Author,

Please check your proof carefully and mark all corrections at the appropriate place in the proof (e.g., by using on-screen annotation in the PDF file) or compile them in a separate list. Note: if you opt to annotate the file with software other than Adobe Reader then please also highlight the appropriate place in the PDF file. To ensure fast publication of your paper please return your corrections within 48 hours.

For correction or revision of any artwork, please consult http://www.elsevier.com/artworkinstructions.

Any queries or remarks that have arisen during the processing of your manuscript are listed below and highlighted by flags in the proof.

\begin{tabular}{|c|c|}
\hline $\begin{array}{l}\text { Location } \\
\text { in article }\end{array}$ & $\begin{array}{l}\text { Query / Remark: Click on the } Q \text { link to find the query's location in text } \\
\text { Please insert your reply or correction at the corresponding line in the proof }\end{array}$ \\
\hline Q1 & Please approve the short title to be used in the running head at the top of each right-hand page. \\
\hline Q2 & $\begin{array}{l}\text { This is how your name will appear on the contributor's list. Please add your academic title and any other } \\
\text { necessary titles and professional affiliations, verify the information, and OK } \\
\text { ZOLTAN CSANADI, MD, PhD, Department of Cardiology, University of Debrecen, Debrecen, Hungary; } \\
\text { Case Western Reserve University, Cleveland, Ohio; Interventional Electrophysiology, Scripps Clinic, San } \\
\text { Diego, California } \\
\text { EDINA NAGY-BALÓ, MD, Department of Cardiology, University of Debrecen, Debrecen, Hungary; } \\
\text { Case Western Reserve University, Cleveland, Ohio; Interventional Electrophysiology, Scripps Clinic, San } \\
\text { Diego, California } \\
\text { STEPHAN DANIK, MD, Case Western Reserve University, Cleveland, Ohio; Interventional } \\
\text { Electrophysiology, Scripps Clinic, San Diego, California; Al-Sabah Arrhythmia Institute (AI), St. Luke's } \\
\text { Hospital, New York } \\
\text { CONOR BARRETT, MD, Case Western Reserve University, Cleveland, Ohio; Interventional } \\
\text { Electrophysiology, Scripps Clinic, San Diego, California; Al-Sabah Arrhythmia Institute (AI), St. Luke's } \\
\text { Hospital, New York } \\
\text { J. DAVID BURKHARDT, MD, Case Western Reserve University, Cleveland, Ohio; Interventional } \\
\text { Electrophysiology, Scripps Clinic, San Diego, California; Texas Cardiac Arrhythmia Institute, St. David's } \\
\text { Medical Center, Austin, Texas } \\
\text { JAVIER SANCHEZ, Case Western Reserve University, Cleveland, Ohio; Interventional } \\
\text { Electrophysiology, Scripps Clinic, San Diego, California; Texas Cardiac Arrhythmia Institute, St. David's } \\
\text { Medical Center, Austin, Texas } \\
\text { PASQUALE SANTANGELI, MD, Case Western Reserve University, Cleveland, Ohio; Interventional } \\
\text { Electrophysiology, Scripps Clinic, San Diego, California; Clinical Cardiac Electrophysiology, University } \\
\text { of Pennsylvania, Philadelphia, Pennsylvania } \\
\text { FRANCESCO SANTORO, MD, Case Western Reserve University, Cleveland, Ohio; Interventional } \\
\text { Electrophysiology, Scripps Clinic, San Diego, California; Department of Cardiology, University of Foggia, }\end{array}$ \\
\hline
\end{tabular}


Foggia, Italy

LUIGI DI BIASE, MD, PhD, Case Western Reserve University, Cleveland, Ohio; Interventional Electrophysiology, Scripps Clinic, San Diego, California; Texas Cardiac Arrhythmia Institute, St. David's Medical Center, Austin, Texas; Department of Cardiology, University of Foggia, Foggia, Italy; Albert Einstein College of Medicine, Montefiore Hospital, Bronx, New York; Department of Biomedical Engineering, University of Texas, Austin, Texas

ANDREA NATALE, MD, Case Western Reserve University, Cleveland, Ohio; Interventional Electrophysiology, Scripps Clinic, San Diego, California; Al-Sabah Arrhythmia Institute (AI), St. Luke's Hospital, New York; Texas Cardiac Arrhythmia Institute, St. David's Medical Center; Department of Biomedical Engineering, University of Texas, Austin, Texas

Q3

Q4

Q5

Q6

Q7

Q8

Q9

Q10

Q11

Q12

Q13

Q14

Are author names and order of authors OK as set?

Please provide professional degrees (e.g., PhD, MD) for the author "Javier Sanchez".

Affiliation: Designators ' 8 and 9' (now b and c) were not cited, hence they have been cited for all authors. Please check and correct if necessary.

The following synopsis was created from the introductory and summary paragraphs of your article, because a separate abstract was not provided. Please confirm OK, or submit a replacement (also less than 100 words). Please note that the synopsis will appear in PubMed: Transcatheter treatment of atrial fibrillation (AF) is a complex intervention performed in patients who are at inherently increased risk of a thromboembolic complication, including stroke. It is therefore not surprising that cerebrovascular accidents have been among the most feared complications since the inception of AF ablation. While improvements have been made to limit the incidence of thromboembolic events during catheter ablation of $\mathrm{AF}$, the optimal strategy to minimize such complications has yet to be determined. It is hoped that larger trials using periprocedural anticoagulation strategies can be undertaken to definitively address these important concerns.

Please verify the affiliation addresses and provide the missing information (department name for affiliations "b, d, e, h"; street name, zip code for affiliations "b-i", city name for affiliation 'd').

If there are any drug dosages in your article, please verify them and indicate that you have done so by initialing this query.

Ref. 1 was not cited in the text, hence it has been placed at the end of the first sentence of the first paragraph. Please verify.

Originally Refs. [13] and [23] were identical, hence the latter has been removed from the reference list and subsequent references have been renumbered.

Please update "in press" details in Ref. 59.

Table 1 column 3: As per editorial remarks "Please check what does the numeral 9 refer to?"

Please verify the citation "Neumann et al, 2011" in Table 1.

As per editorial remarks "Please check disclosure statement and update if necessary."

Please check this box or indicate

your approval if you have no

corrections to make to the PDF file

Thank you for your assistance. 